\title{
THE EXCRETION OF 4-HYDROXY-3-METHOXY-MANDELIC ACID BY CHILDREN
}

\author{
BY \\ T. McKENDRICK* and R. W. H. EDWARDS \\ From The Hospital for Sick Children, Great Ormond Street, London
}

(RECEIVED FOR PUBLICATION NOVEMBER 9, 1964)

Much interest has been shown recently in the urinary excretion of 4-hydroxy-3-methoxy-mandelic acid (HMMA, but also known as VMA). It is accepted that its estimation may assist in the diagnosis of neuroblastoma and phaeochromocytoma, the high excretion usually found with these tumours being well above that possibly due to dietary constitutents, the stress of hospital admission, or recent operation. While most workers in describing methods or investigating clinical groups have included small numbers of normal subjects in order to define normal values of excretion, there have been no systematic studies of the excretion pattern in childhood. This paper records a study of 24-hour HMMA excretion in 132 normal children and in several small groups of children suffering from conditions that have been suspected of raising excretion rates of HMMA. In the series of children with proven neuroblastoma, as in those of most other workers, there are a few with normal HMMA excretion. Some of the non-pathological factors that might influence HMMA excretion have also been studied.

\section{Subjects and Methods}

The following children provided 24-hour specimens of urine:

Group 1: 66 healthy children aged between 1 year 4 months and 15 years 6 months. They were normally active at home. The only restriction imposed was the avoidance of ice-cream and banana for the days before and during collection.

Group 2: 66 children in hospital, 54 of them were between 1 year 1 month and 15 years 10 months, the other 12 being less than 1 year. Though they were in hospital these subjects were active but probably less so than at home. Patients with acute or urinary infections, recent

* Present address: Department of Child Health, Liverpool University, Alder Hey Children's Hospital, Liverpool 12. or imminent operation, or with any renal, endocrine, or metabolic disorder were excluded. The same dietary restrictions as in group 1 were applied.

Group 3: 20 children from group 1 provided a second specimen one year after the first.

Group 4: 21 patients aged from 4 months to 13 years 5 months who, though suspected of an adrenal tumour or neuroblastoma, were subsequently shown to have neither.

Group 5: 7 patients, all in hospital with hypertension from various causes. They were aged from 3 months to 12 years 4 months.

Group 6: 10 patients with tumours other than neuroblastoma, aged from 6 months to 11 years 5 months.

Group 7: 6 patients from whom neuroblastomata had previously been removed or had regressed. They were aged from 11 months to 13 years.

Group 8: 13 patients in whom a neuroblastoma was histologically proven. Their ages ranged from 5 months to 8 years.

From 8 subjects separate samples were collected for successive timed periods of 3 to 5 hours during 24 hours. All subjects passed between 5 and 7 specimens. Of the 8,4 were in hospital but not in bed, and the other 4 were healthy normal subjects.

Successive 24-hour specimens for 1 day before and 3 days after a major operation were collected from 3 subjects. Similar collections were made from 5 subjects who consumed extra diet previously considered to interfere with HMMA estimation.

Urine was collected in polythene bottles containing $1 \mathrm{ml} .5 \mathrm{~N}-\mathrm{HCl}$ per year of age of the child or proportionately less for the non-24 hour collections. Bottles were stored if necessary before analysis at $4^{\circ} \mathrm{C}$. Analyses were performed in batches by the method of Pisano, Crout, and Abraham (1962). Each urine sample was analysed in duplicate with a blank and at least one recovery control of $25 \mu \mathrm{g}$. HMMA* in each batch (of 2 to 5 samples).

\footnotetext{
* Supplied by Koch-Light Laboratories, Colnbrook, Bucks.
} 


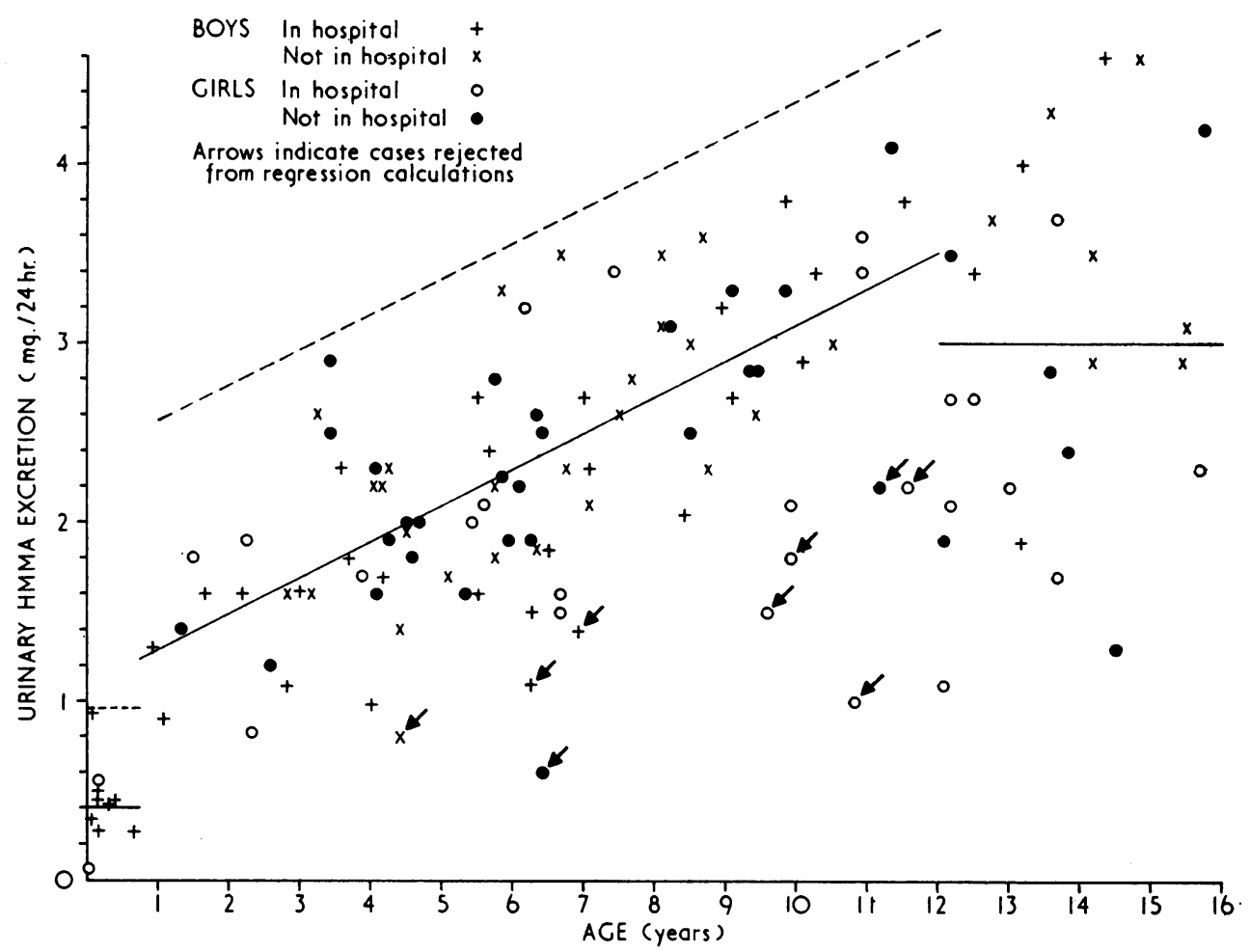

FIG. 1.-Scatter diagram relating 24-hour excretion of HMMA to age. The mean regression line between 1 and 11.9 years is drawn in and $1 \%$ upper limit of confidence .....

Children aged less than 1 year and those aged between 12 and 15.9 years were considered as separate groups. The average and $1 \%$ upper limit are shown in a similar manner to the regression line and its limit.

\section{Results}

The mean recovery by periodate oxidation of aqueous HMMA $25 \mu \mathrm{g} . / \mathrm{ml}$. was $96.8 \pm 3.8 \%$ (S.D.). From urine the mean recovery over the whole process was $84 \cdot 8 \pm 6 \cdot 8 \%$ (S.D.) over 100 consecutive analyses.

The excretion of HMMA by healthy children and those in hospital (groups 1 and 2) is shown graphically in Fig. 1, where male and female, healthy and hospitalized children are separately designated. The regression of excretion on age was calculated for those aged 1 to 11.9 years (96 subjects in all); there were too few subjects below the age of 1 year, and those over the age of 12 showed much greater variation. The lowest points were not included in the calculation of the regression for it seemed likely that they represented incomplete collection or other abnormality; from 5 of 9 subjects thus 'eliminated' unusually small 24-hour urine specimens were received. With this elimination the numbers of points excluded by upper and lower $5 \%$ levels became equal.

The statistical test used to determine the signifi- cance of differences between subgroups of the children was based on a calculation of the regression data and then of the HMMA excretion at a standardized age. The age chosen was that of the average age of all the children and was termed the grand mean age. The average age of the children in each subgroup was very similar to the grand mean age so that no weighting due to age difference occurred. The calculated data is presented in Table 1 .

The differences between the subgroups was tested using ' $t$ ' values, but significance was not reached. This included a comparison of males and females, children in hospital and not in hospital, and those repeated after one year compared with their original values.

From the data of all the children the lines of regression and probability were calculated. Thus Fig. 1 shows the $1 \%$ line which is taken as the upper limit of normality. It is clear from Fig. 1 that the children aged less than 1 year were not proximate to the regression line. Their mean 24-hour HMMA excretion was $0.47 \pm 0.33 \mathrm{mg}$. (SD) at 3 months of age. 
TABLE 1

STATISTICAL DATA CALCULATED FROM HMMA EXCRETION OF NORMAL CHILDREN AGED 1 TO 11.9 YEARS

\begin{tabular}{|c|c|c|c|c|c|c|c|c|c|c|c|}
\hline \multirow{2}{*}{ Group } & \multirow{2}{*}{$\mathbf{n}$} & \multirow{2}{*}{$\overline{\mathbf{x}}$} & \multirow{2}{*}{$\overline{\mathbf{y}}$} & \multirow{2}{*}{$\Sigma \mathrm{x}$} & \multirow{2}{*}{$\Sigma \mathrm{x}^{2}$} & \multirow{2}{*}{$\Sigma \mathrm{xy}$} & \multirow{2}{*}{$\Sigma \mathrm{y}$} & \multicolumn{2}{|c|}{ Regression } & \multirow{2}{*}{$\begin{array}{c}\text { Calculated } \\
\text { HMMA } \\
\text { Excretion } \\
\text { (mg./24 } \\
\text { hr.) at } \\
\text { grand } \\
\text { mean age } \\
\text { Y }\end{array}$} & \multirow{2}{*}{$\begin{array}{c}\text { Stand- } \\
\text { ard } \\
\text { Devia- } \\
\text { tion S }\end{array}$} \\
\hline & & & & & & & & $\begin{array}{c}\text { Slope } \\
\text { (b) }\end{array}$ & $\begin{array}{c}\text { Inter- } \\
\text { cept }\end{array}$ & & \\
\hline $\begin{array}{l}\text { A: males } \\
\text { B: males in hospital } \\
\text { C: females } \\
\text { D: females in hospital } \\
\text { Total in Groups A-D } \\
\text { E: Group } 3\end{array}$ & $\begin{array}{l}25 \\
23 \\
25 \\
14 \\
87 \\
18\end{array}$ & $\begin{array}{l}6 \cdot 28 \\
5 \cdot 99 \\
6 \cdot 02 \\
6 \cdot 52 \\
6 \cdot 17(Y) \\
6 \cdot 66\end{array}$ & $\begin{array}{l}2 \cdot 44 \\
2 \cdot 24 \\
2 \cdot 37 \\
2 \cdot 24 \\
2 \cdot 34 \\
2 \cdot 43\end{array}$ & $\begin{array}{l}156 \cdot 99 \\
137 \cdot 74 \\
130 \cdot 41 \\
91 \cdot 22 \\
536 \cdot 36 \\
119 \cdot 91\end{array}$ & $\begin{array}{r}1084 \cdot 0126 \\
1026 \cdot 8590 \\
1050 \cdot 2780 \\
741 \cdot 2798 \\
3902 \cdot 3794 \\
870 \cdot 379\end{array}$ & $\begin{array}{r}403 \cdot 196 \\
350 \cdot 447 \\
392 \cdot 982 \\
226 \cdot 443 \\
1373 \cdot 068 \\
309 \cdot 799\end{array}$ & $\begin{array}{r}61 \cdot 10 \\
51 \cdot 50 \\
59 \cdot 35 \\
31 \cdot 32 \\
203 \cdot 27 \\
43 \cdot 80\end{array}$ & $\begin{array}{l}0 \cdot 199 \\
0 \cdot 208 \\
0 \cdot 247 \\
0 \cdot 155 \\
0 \cdot 201 \\
0 \cdot 252\end{array}$ & $\begin{array}{l}1 \cdot 20 \\
0 \cdot 99 \\
0 \cdot 89 \\
1 \cdot 25 \\
1 \cdot 09 \\
0 \cdot 75\end{array}$ & $\begin{array}{l}2 \cdot 41 \\
2 \cdot 27 \\
2 \cdot 41 \\
2 \cdot 20 \\
2 \cdot 34 \\
2 \cdot 30\end{array}$ & $\begin{array}{l}0.48 \\
0 \cdot 56 \\
0 \cdot 24 \\
0 \cdot 72 \\
0 \cdot 53 \\
0.62\end{array}$ \\
\hline
\end{tabular}

Note: $\mathrm{n}$ is the number of subjects in each group. Age is designated $\mathrm{x}$ and HMMA excretion $\mathrm{y}, \overline{\mathrm{x}}$ and $\overline{\mathrm{y}}$ being the mean values. $\mathrm{Y}$ is grand mean. Results of 'outliers' indicated on Fig. 1 are not included in these calculations.

The significance of differences between the various pairs of HMMA values at the grand mean age (e.g. (A) and (C), and (B) and (D), (A) and (B) etc.) were tested using $t=$ difference $\left(S_{1} / n_{1}+S_{2} / n_{2}\right)-\frac{1}{2}$ where $n_{1}$ and $n_{2}$ are the numbers of subjects in the groups compared and $S_{1}$ and $S_{2}$ are the standard deviations calculated for each group using $(\mathrm{n}-2) \mathrm{S}=\Sigma(\mathrm{y}-\overline{\mathrm{y}})^{2}-\mathrm{b}^{2} \Sigma(\mathrm{x}-\overline{\mathrm{x}})^{2} ; \mathrm{b}$ is the regression slope.

The relatively wide scatter shown in the children aged 12-15.9 years has made firm conclusions difficult. Study of the volumes of the girls' specimens received suggested that not all were complete 24-hour saves. The over-all average excretion was $3.05 \pm 1.05$ (SD; 25 subjects): that of the 11 boys was $3 \cdot 54 \pm 1 \cdot 64(\mathrm{SD})$ and of the girls $2 \cdot 48 \pm 1 \cdot 43$ (SD).
Brief clinical features and HMMA excretions of patients in groups 4 to 8 are shown in Tables 2 to 6 . With the exception of children with neuroblastoma, none of these groups included any child with HMMA excretion above the $1 \%$ confidence limit of groups 1 and 2.

Of the 13 patients with neuroblastoma (all, as well as those of group 7 being proven histologically), 10

TABLE 2

PRINCIPAL FEATURES AND HMMA EXCRETION IN CHILDREN SUSPECTED OF HAVING NEUROBLASTOMA OR PHAEOCHROMOCYTOMA (GROUP 4)

\begin{tabular}{|c|c|c|c|c|c|c|c|}
\hline Patient & Sex & Age (yr.) & Clinical Features & $\begin{array}{l}\text { Suspected } \\
\text { Diagnosis }\end{array}$ & $\begin{array}{l}\text { Dura- } \\
\text { tion of } \\
\text { History } \\
\text { (mth.) }\end{array}$ & $\begin{array}{l}\text { HMMA } \\
\text { Excretion } \\
\text { (mg./24 } \\
\mathrm{hr} .)\end{array}$ & Conclusion \\
\hline $\begin{array}{l}\text { P.S. } \\
\text { D.W. } \\
\text { S.W. }\end{array}$ & $\begin{array}{c}\mathbf{M} \\
\mathbf{M}\end{array}$ & $\begin{array}{l}3 / 12 \\
7 / 12 \\
10 / 12\end{array}$ & $\begin{array}{l}\text { Oedema, ascites, hypertension } \\
\text { Mass in left loin } \\
\text { Failure to thrive; large liver and } \\
\text { spleen }\end{array}$ & $\begin{array}{l}\text { Neuroblastoma } \\
\text { Neuroblastoma } \\
\text { Neuroblastoma }\end{array}$ & $\begin{array}{l}2 \\
3 \\
5\end{array}$ & $\begin{array}{l}0 \cdot 22 \\
0 \cdot 7 \\
1 \cdot 4\end{array}$ & $\begin{array}{l}\text { Protein-losing enteropathy } \\
\text { Urinary infection } \\
\text { Niemann Pick's disease }\end{array}$ \\
\hline $\begin{array}{l}\text { S.H. } \\
\text { C.M. }\end{array}$ & $\begin{array}{l}\mathbf{F} \\
\mathbf{F}\end{array}$ & $11 / 12$ & $\begin{array}{l}\text { Anaemia, high ESR and PUO } \\
\text { Failure to thrive, anaemia, } \\
\text { abdominal swelling, sweating }\end{array}$ & $\begin{array}{l}\text { Neuroblastoma } \\
\text { Neuroblastoma }\end{array}$ & $\begin{array}{l}1 \\
7\end{array}$ & $\begin{array}{l}1 \cdot 5 \\
0 \cdot 32\end{array}$ & $\begin{array}{l}\text { Recovery but no diagnosis } \\
\text { ? Protein-losing enteropathy }\end{array}$ \\
\hline $\begin{array}{l}\text { G.D. } \\
\text { N.H. } \\
\text { D.B. } \\
\text { T.K. }\end{array}$ & $\begin{array}{l}\mathbf{M} \\
\mathbf{M} \\
\mathbf{F} \\
\mathbf{M}\end{array}$ & $\begin{array}{l}18 / 12 \\
25 / 12 \\
25 / 12 \\
29 / 12\end{array}$ & $\begin{array}{l}\text { Unusual seizures } \\
\text { Large liver } \\
\text { Anaemia; glands in neck } \\
\text { Attacks of syncope ? hypoglycae- } \\
\text { mia }\end{array}$ & $\begin{array}{l}\text { Phaeochromocytoma } \\
\text { Neuroblastoma } \\
\text { Neuroblastoma } \\
\text { Phaeochromocytoma }\end{array}$ & $\begin{array}{r}12 \\
1 \\
2 \\
9\end{array}$ & $\begin{array}{l}1 \cdot 1 \\
0 \cdot 75 \\
1 \cdot 1 \\
1 \cdot 7\end{array}$ & $\begin{array}{l}\text { Epilepsy } \\
\text { Cirrhosis } \\
\text { Infective adenitis } \\
\text { Syncope }\end{array}$ \\
\hline $\begin{array}{l}\text { P.I. } \\
\text { D.H. } \\
\text { D.S. }\end{array}$ & $\begin{array}{l}\mathbf{M} \\
\mathbf{M}\end{array}$ & $\begin{array}{ll}3 & 2 / 12 \\
5 & 1 / 12 \\
5 & 3 / 12\end{array}$ & $\begin{array}{l}\text { PUO and large liver } \\
\text { Failure to thrive; large liver } \\
\text { Pain in back with oedema and } \\
\text { limitation of movement }\end{array}$ & $\begin{array}{l}\text { Neuroblastoma } \\
\text { Neuroblastoma } \\
\text { Neuroblastoma }\end{array}$ & $\begin{array}{r}2 \\
24 \\
\frac{1}{2}\end{array}$ & $\begin{array}{l}0 \cdot 6 \\
2 \cdot 1 \\
2 \cdot 3\end{array}$ & $\begin{array}{l}\text { Leishmaniasis } \\
\text { Malnutrition } \\
\text { Recovery; probably mild } \\
\text { rheumatoid arthritis }\end{array}$ \\
\hline C.P. & $\mathbf{M}$ & $53 / 12$ & $\begin{array}{l}\text { Attacks of fever, sweating, vomit- } \\
\text { ing, and drowsiness }\end{array}$ & Phaeochromocytoma & 24 & $1 \cdot 0$ & Periodic syndrome \\
\hline $\begin{array}{l}\text { V.C. } \\
\text { P.F. }\end{array}$ & $\underset{\mathbf{M}}{\mathbf{F}}$ & $\begin{array}{l}56 / 12 \\
76 / 12\end{array}$ & $\begin{array}{l}\text { Anaemia; decalcified vertebrae } \\
\text { Right hemiparesis and hyper- } \\
\text { tension }\end{array}$ & $\begin{array}{c}\text { Neuroblastoma } \\
\text { Phaeochromocytoma }\end{array}$ & $\begin{array}{l}9 \\
8\end{array}$ & $\begin{array}{l}2 \cdot 1 \\
2 \cdot 8\end{array}$ & $\begin{array}{l}\text { Leukaemia } \\
\text { Post-op. thrombosis }\end{array}$ \\
\hline $\begin{array}{l}\text { B.R. } \\
\text { S.G. } \\
\text { M.H. }\end{array}$ & $\begin{array}{l}\mathbf{M} \\
\mathbf{F}\end{array}$ & $\begin{array}{l}88 / 12 \\
9 \\
99 / 12\end{array}$ & $\begin{array}{l}\text { Fever; mass in abdomen } \\
\text { Exophthalmos; hepatomegaly } \\
\text { Flexion spasm of the hip with } \\
\text { tenderness in pelvis; abdominal }\end{array}$ & $\begin{array}{l}\text { Neuroblastoma } \\
\text { Neuroblastoma } \\
\text { Neuroblastoma }\end{array}$ & $\begin{array}{r}1 \\
1 \\
60\end{array}$ & $\begin{array}{l}1 \cdot 5 \\
2 \cdot 3 \\
2 \cdot 6\end{array}$ & $\begin{array}{l}\text { Nephritis } \\
\text { Not known } \\
\text { Urinary infection }\end{array}$ \\
\hline J.N. & $\mathbf{M}$ & $109 / 12$ & $\begin{array}{l}\text { Dain } \\
\text { headaches, flushing }\end{array}$ & Phaeochromocytoma & 12 & $3 \cdot 4$ & Emotional problem \\
\hline A.W. & $\mathbf{M}$ & $109 / 12$ & $\begin{array}{l}\text { Mentally subnormal, reputed to } \\
\text { improve on cheese }\end{array}$ & $\begin{array}{l}\text { Anomaly of amino } \\
\text { acid metabolism }\end{array}$ & 129 & $2 \cdot 9$ & Not yet diagnosed \\
\hline G.L. & $\mathbf{M}$ & $116 / 12$ & $\begin{array}{l}\text { Recurrent abdominal pain; } \\
\text { hypertension on one occasion }\end{array}$ & Phaeochromocytoma & 3 & $3 \cdot 2$ & No organic lesion found \\
\hline M.S. & $\mathbf{M}$ & 13 & Pain and tumour in right thigh & Neuroblastoma & 11 & $2 \cdot 1$ & $\begin{array}{l}\text { Multiple malignant tumours; } \\
\text { histology indefinite; no } \\
\text { necropsy }\end{array}$ \\
\hline A.L. & $\mathbf{M}$ & $136 / 12$ & Loss of weight; mass in left loin & Neuroblastoma & 5 & $3 \cdot 8$ & Hydronephrosis \\
\hline
\end{tabular}


TABLE 3

PRINCIPAL FEATURES AND HMMA EXCRETION IN CHILDREN WITH HYPERTENSION (GROUP 5)

\begin{tabular}{|c|c|c|c|c|c|c|}
\hline Name & Sex & Age (yr.) & $\begin{array}{c}\text { Approximate Blood } \\
\text { Pressure (mm.Hg) }\end{array}$ & $\begin{array}{l}\text { Duration } \\
\text { (mth.) }\end{array}$ & Cause & $\underset{\text { Excretion }}{\text { HMM. } / 24 \mathrm{hr} .)}$ \\
\hline $\begin{array}{l}\text { G.H. } \\
\text { O.H. }\end{array}$ & $\underset{\mathbf{M}}{\mathbf{F}}$ & $\begin{array}{l}3 / 12 \\
4 / 12\end{array}$ & $\begin{array}{l}125 / 90 \text { to } 180 / 120 \\
90 / 60 \text { to } 120 / 90\end{array}$ & ?3 & $\begin{array}{l}\text { Coarctation of aorta } \\
\text { ? Hypoplastic rt. kidney }\end{array}$ & $\begin{array}{l}0 \cdot 30 \\
0 \cdot 43\end{array}$ \\
\hline $\begin{array}{l}\text { A.H. } \\
\text { G.W. } \\
\text { D.M. } \\
\text { P.C. } \\
\text { S.V. }\end{array}$ & $\begin{array}{c}\mathbf{M} \\
\mathbf{F} \\
\mathbf{M} \\
\mathbf{M}\end{array}$ & $\begin{array}{cc} & 7 / 12 \\
5 & 6 / 12 \\
9 & \\
11 & \\
12 & 6 / 12\end{array}$ & $\begin{array}{l}120 / 80 \text { to } 170 / 130 \\
190 / 50 \\
150 / 80 \text { to } 200 / 140 \\
160 / 120 \\
260 / 200\end{array}$ & $\begin{aligned} & 12^{\frac{1}{2}} \\
&> 3 \\
&> 1 \frac{1}{2} \\
& 120\end{aligned}$ & $\begin{array}{l}\text { ? Polyarteritis } \\
\text { Chronic nephritis } \\
\text { Chronic pyelonephritis } \\
\text { Chronic pyelonephritis } \\
\text { Renal disease secondary } \\
\text { to hypercalcaemia }\end{array}$ & $\begin{array}{l}0 \cdot 82 \\
1 \cdot 8 \\
2 \cdot 75 \\
3 \cdot 2 \\
1 \cdot 2\end{array}$ \\
\hline
\end{tabular}

TABLE 4

HMMA EXCRETION IN CHILDREN WITH TUMOURS NOT ORIGINATING FROM SYMPATHETIC NERVOUS TISSUE (GROUP 6)

\begin{tabular}{|c|c|c|c|c|}
\hline Name & Sex & Age (yr.) & Tumour & $\underset{\text { Excretion }}{\text { HMMA }}$ \\
\hline $\begin{array}{l}\text { J.G. } \\
\text { P.S. } \\
\text { A.N. } \\
\text { J.W. } \\
\text { L.W. } \\
\text { A.B. } \\
\text { P.B. } \\
\text { M.M. } \\
\text { B.L. } \\
\text { S.G. }\end{array}$ & $\begin{array}{l}\mathbf{M} \\
\mathbf{M} \\
\mathbf{M} \\
\mathbf{F} \\
\mathbf{F} \\
\mathbf{M} \\
\mathbf{M} \\
\mathbf{F} \\
\mathbf{M} \\
\mathbf{M}\end{array}$ & $\begin{array}{ll} & 6 / 12 \\
6 / 12 \\
10 / 12 \\
46 / 12 \\
46 / 12 \\
5 \\
6 \\
9 \\
104 / 12 \\
116 / 12\end{array}$ & $\begin{array}{l}\text { Reticulum cell sarcoma } \\
\text { Hepatoblastoma } \\
\text { Reticulum cell sarcoma } \\
\text { Lymphosarcoma } \\
\text { Malignant hepatoblastoma } \\
\text { Nephroblastoma } \\
\text { Fibrosarcoma } \\
\text { ? Angio-sarcoma } \\
\text { Ganglioneuroma of coccyx }\end{array}$ & $\begin{array}{l}0 \cdot 51 \\
0 \cdot 85 \\
0 \cdot 50 \\
2 \cdot 2 \\
2 \cdot 9 \\
1 \cdot 6 \\
2 \cdot 6 \\
3 \cdot 0 \\
1 \cdot 5 \\
4 \cdot 0\end{array}$ \\
\hline
\end{tabular}

TABLE 5

PRINCIPAL FEATURES AND HMMA EXCRETION IN CHILDREN FROM WHOM A NEUROBLASTOMA HAD BEEN REMOVED OR IN WHOM REGRESSION WAS CLINICALLY COMPLETE (GROUP 7)

\begin{tabular}{|c|c|c|c|c|c|c|c|}
\hline Name & Sex & $\underset{(y r .)}{\text { Age at }}$ & $\begin{array}{c}\text { Age at } \\
\text { Diagnosis } \\
\text { (yr.) }\end{array}$ & Site & $\begin{array}{l}\text { Duration } \\
\text { of History } \\
\text { (mth.) }\end{array}$ & Present State & $\begin{array}{c}\text { HMMA } \\
\text { Excretion } \\
\text { (mg./24 hr.) }\end{array}$ \\
\hline $\begin{array}{l}\text { T.G. } \\
\text { J.F. } \\
\text { S.E. } \\
\text { C.L.* } \\
\text { S.P.* } \\
\text { A.C. }\end{array}$ & $\begin{array}{l}\mathbf{F} \\
\mathbf{F} \\
\mathbf{F} \\
\mathbf{F} \\
\mathbf{F} \\
\mathbf{F}\end{array}$ & $\begin{aligned} 17 / 12 \\
49 / 12 \\
69 / 12 \\
10 \\
116 / 12 \\
13\end{aligned}$ & $\begin{array}{r}8 / 12 \\
7 / 12 \\
9 / 12 \\
13 / 12 \\
9 / 12 \\
86 / 12\end{array}$ & $\begin{array}{l}\text { Mediastinum } \\
\text { Mediastinum } \\
\text { Left loin } \\
\text { Posterior mediastinum } \\
\text { Upper mediastinum and neck } \\
\text { Left leg and skull }\end{array}$ & $\begin{array}{c}3 \\
\text { About } 1 \\
\frac{1}{2} \\
3 \\
\text { About } 1 \\
6\end{array}$ & $\begin{array}{l}\text { Healthy } \\
\text { Paraplegia } \\
\text { Healthy } \\
\text { Paraplegia } \\
\text { Healthy } \\
\text { Healthy }\end{array}$ & $\begin{array}{l}0 \cdot 20 \\
1 \cdot 8 \\
1 \cdot 3 \\
0 \cdot 32 \\
3 \cdot 7 \\
2 \cdot 3\end{array}$ \\
\hline
\end{tabular}

* These patients were treated medically only because the tumours could not be removed.

TABLE 6

PRINCIPAL FEATURES AND HMMA EXCRETION IN 13 CHILDREN WITH NEUROBLASTOMA (GROUP 8)

\begin{tabular}{|c|c|c|c|c|c|c|c|}
\hline Patient & Sex & Age (yr.) & Clinical Features & $\begin{array}{l}\text { Duration } \\
\text { of History } \\
\text { (mth.) }\end{array}$ & $\begin{array}{l}\text { Abnormal } \\
\text { I.V.P. }\end{array}$ & $\underset{\text { (mg./24 hr.) }}{\text { Excretion }}$ & $\underset{\text { (mth.) }}{\text { Survival }}$ \\
\hline $\begin{array}{l}\text { R.K. } \\
\text { P.G. } \\
\text { J.S. } \\
\text { G.W. } \\
\text { M.G. } \\
\text { J.W. } \\
\text { A.D. } \\
\text { L.S. } \\
\text { P.M. }\end{array}$ & $\begin{array}{l}\mathbf{F} \\
\mathbf{M} \\
\mathbf{M} \\
\mathbf{M} \\
\mathbf{M} \\
\mathbf{F} \\
\mathbf{M} \\
\mathbf{M} \\
\mathbf{M}\end{array}$ & $\begin{array}{ll}1 & 4 / 12 \\
1 & 1 / 12 \\
1 & 1 / 12 \\
1 & 2 / 12 \\
1 & 4 / 12 \\
1 & 4 / 12 \\
2 & \\
2 & 6 / 12 \\
3 & 9 / 12\end{array}$ & $\begin{array}{l}\text { Hepatomegaly } \\
\text { Anaemia; multiple tumours } \\
\text { Cranial mass } \\
\text { Sacral mass } \\
\text { Retroperitoneal mass } \\
\text { Anaemia; retroperitoneal mass } \\
\text { Anaemia; limpness; retroperitoneal mass } \\
\text { Anaemia; proptosis; retroperitoneal mass } \\
\text { Anaemia and PUO; abdominal pain; }\end{array}$ & $\begin{array}{l}3 \\
\frac{1}{4} \\
1 \\
6 \\
\frac{1}{2} \\
12 \\
1 / \frac{3}{4} \\
3\end{array}$ & $\begin{array}{l}\text { N.P. } \\
\text { N.P. } \\
\text { N.P. } \\
\text { N.P. } \\
+ \\
+ \\
+ \\
+ \\
+ \\
+\end{array}$ & $\begin{array}{r}25 \\
63 \\
6 \cdot 5 \\
1 \cdot 3 \\
5 \cdot 3 \\
16 \cdot 0 \\
1 \cdot 1 \\
3 \cdot 1 \\
27 \cdot 5\end{array}$ & $\begin{array}{c}>6 \\
>15 \\
9^{\frac{1}{2}} \\
9^{\frac{3}{4}} \\
14^{\frac{1}{2}} \\
3^{\frac{1}{2}} \\
14\end{array}$ \\
\hline $\begin{array}{l}\text { J.P. } \\
\text { P.E. } \\
\text { B.G. } \\
\text { D.G. }\end{array}$ & $\begin{array}{l}\mathbf{F} \\
\mathbf{M} \\
\mathbf{M}\end{array}$ & $\begin{array}{l}39 / 12 \\
6 \\
7 \\
8\end{array}$ & $\begin{array}{l}\text { Retroperitoneal mass } \\
\text { Paraplegia; pleural effusion; hepatomegaly } \\
\text { Anaemia and PUO } \\
\text { Spinal compression; abdominal mass }\end{array}$ & $\begin{array}{c}2 \\
24 \\
1 \\
9\end{array}$ & $\begin{array}{l}\text { N.P. } \\
\text { N.P. } \\
- \\
-\end{array}$ & $\begin{array}{r}2 \cdot 0 \\
3 \cdot 5 \\
102 \\
192\end{array}$ & $\begin{array}{r}4 \\
27 \\
7 \\
17\end{array}$ \\
\hline
\end{tabular}

I.V.P. = intravenous pyelogram, $+=$ abnormality demonstrated, $-=$ normal, N.P. = 'not performed'. 


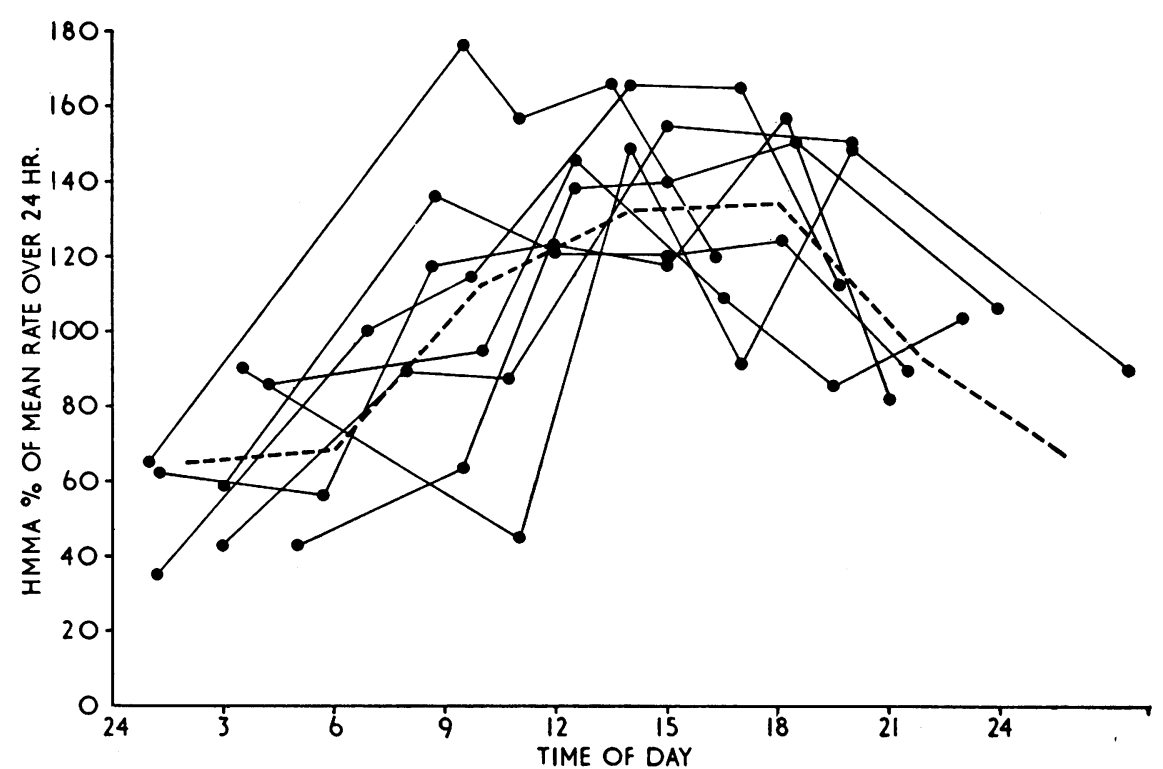

FIG. 2.-Rate of excretion of HMMA throughout 24 hours in 8 subjects, 7 aged between 1 year 4 months and 13 years, and 1 aged 37 years. Rates are plotted as percentage of mean rate over the 24 hours; all subjects produced between 5 and 7 specimens during this period. The curve joining average points was constructed by taking the average of rates of excretion interpolated at four-hourly intervals.

showed the expected high excretion; 3 (G.W., J.P., A.D.) showed a normal excretion, 1 of them showing this on three occasions (J.P.: 2.9, 1.0, and 1.3) but surgical intervention prevented repetition in the others. Two more had levels just inside (P.E.) or not far above (L.S.) the $1 \%$ confidence limits. In several patients, repeated estimations have been made during therapy: P.G. (over 9 months) showed 63, 55, 75 , 48 mg. $/ 24$ hr.; B.G. (over 7 months) showed 102, 187, 137, 116, 102, 147, 93, $128 \mathrm{mg}$./24 hr.; and J.W. (over 8 months) showed 16, 18, 31, $16 \mathrm{mg} . / 24 \mathrm{hr}$.

Fig. 2 shows the changes in relative rate of HMMA excretion in 8 subjects over single 24-hour periods starting at midnight. In order to make the 8 sets of data comparable with each other the results of each set are plotted as a percentage of the mean rate shown over the whole 24-hour period. There is a very marked difference between day and night excretion rates, the average change being from about $65 \%$ of the over-all daily rate at 2.00 a.m. to $135 \%$ between 2.00 and 6.00 p.m.

Three patients were studied before and after major operations. The levels of successive 24-hour excretions are shown in Table 7. Chromatographic and some semi-quantitative methods of HMMA estimation may suffer interference from certain dietary constituents (Robinson, Ratcliffe, and Smith, 1959; Gitlow, Mendlowitz, Khassis, Cohen, and Sha, 1960; Mahler and Humoller, 1962). Substances containing vanillin, e.g. custard, ice-cream, and vanilla essence, and those such as banana and walnut which contain serotonin, noradrenaline, and dopamine have usually been excluded from diets. Five subjects consumed various quantities of these substances (as well as their normal diets) during the second of 3 or 4 successive daily 24-hour urine collections. The excretions of HMMA by these subjects are shown in Table 8 . No increase was demonstrated in any subject.

\section{Discussion}

First isolated by Armstrong, McMillan, and Shaw (1957), HMMA was shown by a study of the fate of tritium-labelled adrenaline and noradrenaline to be a major metabolite of these adrenal medullary secretions (LaBrosse, Axelrod, and Kety, 1958; Kopin, 1960). Most workers have studied the excretion of HMMA in relation to the diagnosis of phaeochromocytoma (e.g. Gitlow et al., 1960), when adrenal medullary activity is known to be excessive, or of neuroblastoma (e.g. von Studnitz, Käser, and Sjoerdsma, 1963; Voorhess and Gardner, 1961). Attempts to study adrenal medullary response to stress by estimating serial HMMA excretion have been reported (Berman and Pettitt, 1961), and observations on other factors such as diurnal variation (Sunderman, Cleveland, Law, and Sunderman, 1960) and diet (Sandler and Ruthven, 1961) have been made. 
TABLE 7

DAILY HMMA EXCRETION OF 3 PATIENTS BEFORE AND AFTER MAJOR OPERATION

\begin{tabular}{|c|c|c|c|c|c|c|}
\hline \multirow{3}{*}{ Patient } & \multirow{3}{*}{ Sex } & \multirow{3}{*}{ Age (yr.) } & \multicolumn{4}{|c|}{ HMMA Excretion (mg./24 hr.) } \\
\hline & & & \multirow{2}{*}{ Pre-operative } & \multicolumn{3}{|c|}{ Post-operative } \\
\hline & & & & 1st day & 2nd day & 3rd day \\
\hline $\begin{array}{l}\text { C.A. } \\
\text { D.S. } \\
\text { D.H. }\end{array}$ & $\begin{array}{c}\mathbf{F} \\
\mathbf{M} \\
\mathbf{F}\end{array}$ & $\begin{array}{l}4 \\
6 \\
8\end{array}$ & $\begin{array}{l}1.6 \\
1.9 \\
2.7\end{array}$ & $\begin{array}{l}1 \cdot 1 \\
2 \cdot 3 \\
2 \cdot 3\end{array}$ & $\begin{array}{l}1 \cdot 1 \\
2 \cdot 1 \\
2 \cdot 7\end{array}$ & $\begin{array}{l}1 \cdot 0 \\
2 \cdot 1\end{array}$ \\
\hline
\end{tabular}

TABLE 8

DAILY HMMA EXCRETION BY SOME SUBJECTS BEFORE AND AFTER CONSUMPTION OF CERTAIN FOODS

\begin{tabular}{|c|c|c|c|c|c|c|c|}
\hline \multirow{3}{*}{ Subject } & \multirow{3}{*}{$\operatorname{Sex}$} & \multirow{3}{*}{ Age (yr.) } & \multirow{3}{*}{ Dietary Extras } & \multicolumn{4}{|c|}{ HMMA Excretion (mg./24 hr.) } \\
\hline & & & & \multirow{2}{*}{ Pre-diet } & \multicolumn{3}{|c|}{ Post-diet } \\
\hline & & & & & 1st day & 2nd day & 3rd day \\
\hline $\begin{array}{l}\text { I.M. } \\
\text { R.S. } \\
\text { G.S. } \\
\text { V.E. } \\
\text { T.M. }\end{array}$ & $\begin{array}{l}\mathbf{M} \\
\mathbf{M} \\
\mathbf{M} \\
\mathbf{F} \\
\mathbf{M}\end{array}$ & $\begin{array}{r}6 \\
8 \\
10 \\
19 \\
37\end{array}$ & $\begin{array}{l}5 \text { bananas; } 2 \text { oz. ice-cream } \\
5 \text { bananas } \\
5 \text { bananas } \\
6 \text { bananas; } 2 \text { oz. walnuts } \\
2 \text { oz. walnuts; } 4 \text { oz. ice-cream }\end{array}$ & $\begin{array}{l}2 \cdot 7 \\
2 \cdot 7 \\
2 \cdot 7 \\
4 \cdot 3 \\
5 \cdot 8\end{array}$ & $\begin{array}{l}2 \cdot 7 \\
2 \cdot 6 \\
2 \cdot 7 \\
3 \cdot 8 \\
6 \cdot 0\end{array}$ & $\begin{array}{l}2 \cdot 6 \\
2 \cdot 8 \\
1 \cdot 8^{*} \\
3 \cdot 9 \\
5 \cdot 4\end{array}$ & $\begin{array}{l}2 \cdot 7 \\
3 \cdot 4 \\
5 \cdot 3\end{array}$ \\
\hline
\end{tabular}

* 12-hour daytime specimen only.

About a dozen methods of estimation have been described. The original method (Armstrong et al., 1957) was by initial extraction with ethyl acetate, followed by two-dimensional chromatography, diazotization, and spectrophotometry; besides taking about 24 hours, this method requires dietary restriction to minimize interference from exogenous phenolic acids. The high voltage electrophoresis methods (von Studnitz and Hanson, 1959; Randrup, 1962) require expensive apparatus and may not always achieve clear separation of different phenolic acids. Low voltage electrophoresis (Klein and Chernaik, 1961) is a prolonged procedure. The diazotization methods of Woiwod and Knight (1961) and Gitlow et al. (1960) tend to give high results, probably because they are less specific. Similarly the colorimetric method using 4-chloro-o-phenylenediamine and phosphoric acid (Fellman, 1963), though conveniently rapid, suffers interference from di-hydroxy-mandelic acid and p-hydroxy-mandelic acid, both of which are found in urine.

Several methods utilize the conversion of HMMA to vanillin as the basis of estimation. In the first of these (Sandler and Ruthven, 1959), initial extraction is by adsorption on to an anion exchange resin. This is followed by elution with ethyl acetate, evaporation to dryness, autoclaving with acid, and finally colorimetric estimation. The procedure is tedious and permits a recovery of $70 \%$ at best. Sunderman et al. (1960) used florisil adsorption for purification. Following extraction by ethyl acetate and back-extraction into potassium carbonate,
HMMA is oxidized by ferricyanide to vanillin; the reaction, however, is less specific, allowing interference from dietary substances, and the terminal colorimetric estimation is sensitive to time, moisture, and temperature. The method described by Pisano et al. (1962) is rapid (less than 3 hours) and involves extraction with ethyl acetate, back-extraction into potassium carbonate, oxidation by sodium periodate, extraction of the resultant vanillin by toluene, and finally spectrophotometric estimation in potassium carbonate. This is the procedure we have employed.

In the present investigation a significant sex difference in HMMA excretion by normal children between the ages of 1 and $11 \cdot 9$ years was not observed. In this age-group there was a good linear relation between excretion and age.

In the first year of life, though the number of observations was small, the scatter was less and it was clear that the points were not co-linear with the older age-group. After the age of 12 years there was much greater variation, particularly in girls, and a sex difference previously reported in adults (Weise, McDonald, and LaBrosse, 1961; Georges and Whitby, 1964) was evident. The over-all average after the age of 12 was $3.05 \pm 1.04$ (SD) and is very similar to the adult level quoted by Ruthven (1963) of $3.7 \pm 1 \cdot 1$ (SD) but is below that stated by Georges and Whitby (1964) of $5.45 \pm 1.05$ (SD).

The marked diurnal variation (from a minimum of $40 \%$ of the mean 24 -hour rate to a maximum of $168 \%$ in one child) was much more pronounced than that 
reported by Weise et al. (1961). It is imperative to collect 24-hour samples, especially if excretion levels near the $1 \%$ 'borderline' are encountered. This variation also makes it difficult to study short-term physiological changes in adrenal medullary activities by serial estimations of HMMA excretion.

The constancy of results following ingested walnut, banana, and vanilla-flavoured ice-cream confirms that excreted HMMA is not of dietary origin and that dietary restriction is unnecessary when collecting urine for estimation by this method.

Residence in hospital per se produced no increase in HMMA excretion, but this observation must be qualified by the fact that no attempt was made to study excretion during the first day of admission; it is possible that the stress of admission might be associated with a temporary rise in HMMA excretion.

The failure to demonstrate a rise in excretion following operative stress is difficult to reconcile with the observations of Hunter, Marshall, and Oram (1963) that operation was followed by a large increase in adrenaline and noradrenaline excretion, and with the demonstration of LaBrosse, Axelrod, Kopin, and Kety (1961) that about $30-40 \%$ of injected adrenaline and noradrenaline could be recovered as HMMA within 48 hours. The latter workers showed that about $5 \%$ of injected adrenaline was excreted unchanged. The daily urinary excretion of adrenaline is quoted as $2-5 \mu \mathrm{g}$. and of noradrenaline as $25-50 \mu \mathrm{g}$. (von Euler, 1956). These figures suggest a daily production of about $0 \cdot 5-1 \cdot 0$ mg. of these substances and if the metabolic pathways were the same this would produce about $0 \cdot 2$ $0.4 \mathrm{mg}$. HMMA daily. Normal HMMA excretion (in adults) is about ten times this value. It may be that the very small increase that results from operative stress is hidden in the relatively larger amount of HMMA from other sources.

Of the 13 histologically proven neuroblastoma cases, 3 failed to show the significantly high HMMA excretion. Such normal excretion does not exclude the diagnosis. Rather does it indicate the need to estimate the homovanillic acid (HVA) excretion. This is unfortunately tedious, but may well be helpful, especially if histological diagnosis from biopsy material is uncertain (e.g. if necrosis is prominent). To the best of the authors' knowledge, patients with neuroblastoma do not have both normal HMMA and normal HVA excretion (M. Sandler, 1963, personal communication; Käser, Bettex, and von Studnitz, 1964).

All the children who remained free of clinical evidence of recurrence following successful surgical removal of neuroblastomata showed normal HMMA excretion. It remains to be seen whether a rise in urinary HMMA excretion precedes clinical effects if further tumours appear. Similarly, continuously high HMMA excretion during therapy provides an indication of the failure of therapy. With the known tendency of this tumour to go into remission, a fall in excretion in a single case might not necessarily indicate therapeutic success.

\section{Summary}

The procedure of Pisano et al. (1962) for the determination of 4-hydroxy-3-methoxy-mandelic acid (HMMA, also known as VMA) has been employed to confirm the high excretion of this catecholamine metabolite in the urine of most children with neuroblastoma.

The urinary excretion of HMMA by 87 normal children was estimated. These children showed a linear regression of HMMA excretion against age within the limit 1 to 12 years of age. The upper $1 \%$ probability level of the regression was taken as the upper limit of normality in assessing the high excretions.

Residence of the children in hospital rather than at home, sex, the presence of tumours other than neuroblastomata, post-operative stress, and dietary factors have no effect on HMMA excretion.

A marked diurnal rhythm of HMMA excretion was demonstrated, making it imperative to perform the determinations only on complete 24-hour urine specimens.

We wish to thank the physicians and surgeons of The Hospital for Sick Children for allowing us to study their patients and Dr. M. D. Baber of the Edgware General Hospital for patient P.G.; Dr. B. E. Clayton for facilities in her department; Dr. C. O. Carter for help in designing the statistical analysis; Mr. R. Gardner and Miss A. Malone for some technical assistance; and the many normal subjects and their parents for producing the samples. One of us (R.W.H.E.) is in receipt of a grant from the Joint Research Board of The Hospital for Sick Children, and the Institute of Child Health.

\section{REFERENCES}

Armstrong, M. D., McMillan, A., and Shaw, K. N. F. (1957). 3-methoxy-4-hydroxy-mandelic acid, a urinary metabolite of norepinephrine. Biochim. biophys. Acta (Amst.), 25, 422.

Berman, M. L., and Pettitt, J. A. (1961). Urinary excretion of 3-methoxy-4-hydroxymandelic acid after several stress situations. J. Lab. clin. Med., 57, 126.

von Euler, U. S. (1956).' Noradrenaline, p. 166. Thomas, Springfield, Illinois.

Fellman, J. H. (1963). Personal communication to C. R. J. Ruthven quoted in The Clinical Chemistry of the Monoamines, ed. $\mathrm{H}$. Varley and A. H. Gowenlock, p. 40. Elsevier, Amsterdam.
V. metabolites of catecholamines in normal individuals and hyper-
metion tensive patients. J. clin. Path., 17, 64.

Gitlow, S. E., Mendlowitz, M., Khassis, S., Cohen, G., and Sha, J. (1960). The diagnosis of pheochromocytoma by determination of urinary 3-methoxy, 4-hydroxymandelic acid. J. clin. Invest., 
Hunter, R. B., Marshall, P. B., and Oram, F. J. (1963), Catechol amine excretion in normal persons and in cases of phaeochromocytoma. Quart. J. Med., 32, 225.

Käser, H., Bettex, M., and Studnitz, W. v. (1964). Further observations on the determination of catecholamine metabolites in tumours of sympathetic nervous system. Arch. Dis. Childh., 39, 168.

Klein, D., and Chernaik, J. M. (1961). Determination of urinary 3-methoxy-4-hydroxymandelic acid (vanillylmandelic acid) by paper electrophoresis. Clin. Chem., 7, 257.

Kopin, I. J. (1960). Technique for the study of alternative metabolic pathways: epinephrine metabolism in man. Science, 131, 1372.

LaBrosse, E. H., Axelrod, J., and Kety, S. S. (1958). O-methylation, the principal route of metabolism of epinephrine in man. ibid., $128,593$.

Lopin, I. J., and Kety, S. S. (1961). Metabolism of 7- $\mathrm{H}^{3}$-epinephrine-d-bitartrate in normal young men. $J$. clin. Invest., 40, 253.

Mahler, D. J., and Humoller, F. L. (1962). A comparison of methods for determining catechol amines and 3-methoxy-4-hydroxymandelic acid in urine. Clin. Chem., 8, 47.

Pisano, J. J., Crout, J. R., and Abraham, D. (1962). Determination of 3-methoxy-4-hydroxymandelic acid in urine. Clin. chim. Acta, 7,285

Randrup, A. (1962). Determination of urinary 3-methoxy-4hydroxy-mandelic (vanilmandelic) acid by electrophoresis at low pH. Scand. J. clin. Lab. Invest., 14, 262

Robinson, R., Ratcliffe, J., and Smith, P. (1959). A screening test for phaeochromocytoma. J. clin. Path., 12, 541 .
Ruthven, C. R. J. (1963). The determination of metabolites of catecholamines by other techniques. In The Clinical Chemistry of the Monoamines, ed. H. Varley and A. H. Gowenlock, p. 39. Elsevier, Amsterdam.

Sandler, M., and Ruthven, C. R. J. (1959). Quantitative colorimetric method for estimation of 3-methoxy-4-hydroxymandelic acid in urine; value in diagnosis of phaeochromocytoma. Lancet, 2, 114 and 1034 .

and (1961). The estimation of 4-hydroxy-3-methoxy, mandelic acid in urine. Biochem. $J .80,78$.

von Studnitz, W., and Hanson, A. (1959). Determination of 3methoxy-4-hydroxymandelic acid in urine by high voltage paper electrophoresis. Scand. J. clin. Lab. Invest., 11, 101.

_- Käser, H., and Sjoerdsma, A. (1963). Spectrum of catechol amine biochemistry in patients with neuroblastoma. New Engl. J. Med., 269, 232

Sunderman, F. W., Jr., Cleveland, P. D. Law, N. C , and Sunderman, F. W. (1960). A method for the determination of 3-methoxy-4hydroxymandelic acid ('vanilmandelic acid') for the diagnosis of pheochromocytoma. Amer. J. clin. Path., 34, 293.

Voorhess, M. L., and Gardner, L. I. (1961). Urinary excretion of norepinephrine, epinephrine and 3-methoxy-4-hydroxymandelic acid by children with neuroblastoma. J. clin. Endocr., 21, 321.

Weise, V. K., McDonald, R. K., and LaBrosse, E. H. (1961). Determination of urinary 3-methoxy-4-hydroxymandelic acid in man. Clin. chim. Acta, 6, 79

Woiwod, A. J., and Knight, R. (1961). The determination of 3-methoxy 4-hydroxy mandelic acid in urine. J. clin. Path., 14, 502 . 